\title{
Características dinâmicas de movimentos seleccionados do basquetebol
}

\author{
Fernanda M. Acquesta \\ Germano M. Peneireiro \\ Roberto Bianco \\ Alberto C. Amadio \\ Júlio C. Serrão
}

Laboratório de Biomecânica

Escola de Educação Física e Esporte

Universidade de São Paulo

https://doi.org/10.5628/rpcd.07.02.174

\section{RESUMO}

O objectivo do estudo se pautou em determinar as características de parâmetros das forças de reacção do solo (FRS) em movimentos típicos do basquetebol executados por jogadores profissionais. Também se objectivou determinar correlações entre o desempenho nos saltos e sua carga mecânica durante as aterrissagens. Oito atletas realizaram cinco tentativas em seis movimentos: arremesso em suspensão (jump), salto com contramovimento (jump \& reach), bandeja, rebote e corrida com bola rápida $(\mathrm{v}=6 \mathrm{~m} / \mathrm{s})$ e lenta $(\mathrm{v}=4 \mathrm{~m} / \mathrm{s})$. Os parâmetros dinâmicos da FRS foram colectados através de uma plataforma de força (KISTLER AG 9287). Os resultados do primeiro pico da força vertical na aterrissagem, do tempo para o para o pico e do impulso foram: no jump 4,54 $\pm 1,10$ PC, 0,08 \pm 0,01s, 0,13 \pm 0,04 PC.s; no rebote $5,39 \pm 1,26$ PC, $0,07 \pm 0,01 \mathrm{~s}, 0,18 \pm$ 0,04 PC.s; e na bandeja 7,27 $\pm 2,71$ PC, $0,08 \pm 0,13 \mathrm{~s}, 0,24 \pm$ 0,04 PC.s. Os resultados evidenciam que o aparelho locomotor é submetido a uma carga mecânica relativamente alta durante os movimentos. As baixas correlações entre o desempenho do salto e o primeiro pico da força vertical na aterrissagem $(0,44$ no jump; 0.22 no rebote; e 0.34 na bandeja) sugerem que o desempenho do salto não tem influência determinante na carga gerada.

Palavras-chave: biomecânica, basquetebol, força de reacção do solo, carga, desempenho

\begin{abstract}
Dynamical characteristics of basketball specific movements

The purpose of this study was to evaluate dynamical parameters of the ground reaction force (GRF) in specific basketball movements performed by Brazilian professional players. It was also aimed to determine correlations between jump performance and its impact shock during landings. Eight athletes performed 5 trials in each movement: jump shot, jump and reach, layup, rebound and dribble at two different speeds (control: $v=4 \mathrm{~m} / \mathrm{s}$ and speed: $v=6 \mathrm{~m} / \mathrm{s}$ ). Dynamical parameters of GRF were sampled by a force platform (KISTLER AG 9287).

Results of the first peak of vertical force in landing, peak rise time and impulse were, respectively: in jump shot $4.54 \pm 1.10 \mathrm{BW}, 0.08 \pm$ 0.01 s, and $0.13 \pm 0.04 \mathrm{BW} . \mathrm{s}$; in rebound $5.39 \pm 1.26 \mathrm{BW}, 0.07 \pm$ $0.01 \mathrm{~s}$, and $0.18 \pm 0.04 \mathrm{BW} . \mathrm{s} ;$ and in layup $7.27 \pm 2.71 \mathrm{BW}, 0.08$ $\pm 0.13 \mathrm{~s}$, and $0.24 \pm 0.04$ BW.s. These results showed that the player's body is submitted to a relatively high impact shock during basketball movements. The low correlations between jump high and the first peak of vertical force ( $r=0.44$ in jump shot, $r=0.22$ in rebound, and $r=0.34$ in layup) demonstrated that jump performance did not have a relevant influence on impact shock during landings.
\end{abstract}

Key-words: biomechanics, basketball, ground reaction force, load, performance. 


\section{INTRODUÇÃO}

O basquetebol é uma modalidade esportiva que envolve corridas, saltos, mudanças de direcção. Tais movimentos condicionam a geração de cargas externas, cuja aplicação está relacionada com o surgimento de lesões por estresse em modalidades esportivas como a corrida $(17,29,26,9)$. No entanto, essa relação ainda não foi bem determinada para o basquetebol. Apesar da popularidade do basquetebol, raros são os estudos que abordam questões relativas aos parâmetros mecânicos que regem os movimentos típicos da modalidade. Como exemplo, tem-se o estudo de McClay et al. (18) e de Miller e Bartlett (22), que abordam características cinemáticas dos movimentos usuais do basquetebol. Dados relativos a parâmetros eletromiográficos são raros, bem como aqueles que descrevem os parâmetros dinâmicos dos movimentos, como o trabalho de McClay et al. (19).

Torna-se interessante observar que apesar do pequeno número de estudos que caracterizam as respostas dinâmicas, e que em ultima instância poderiam contribuir no entendimento da relação entre a carga mecânica e seu potencial lesivo, muitos são os estudos que descrevem as lesões mais comuns na modalidade.

Segundo Henry et al. (11), Zelisko et al. (33), Colliander et al. (8), Apple (5), Iwamoto e Takeda (13), Arena (4), Harmer (10), Junge et al. (15) e Major (16), os estudos apontam uma prevalência das lesões no basquetebol nas extremidades inferiores. $\mathrm{O}$ joelho e tornozelo são as articulações mais frequentemente lesadas por torções, luxações e tendinites. Os pés, especificamente o quinto metatarso, são os mais acometidos por fracturas por estresse. Segundo esses estudos, a maioria das lesões no basquetebol, está associada ao excesso de treinamento, condicionado pela combinação entre a intensidade e o volume em que os movimentos são realizados. Haas ${ }^{1}$ apud McClay et al. (19) reportou, no período entre 1983 e 1987, um total de 44 lesões em 36 jogadores da liga profissional norte-americana de basquete. Estimou-se uma incidência de uma fractura por estresse a cada 32 jogadores. Zelisko et al. (33) apontam um índice de $0,7 \%$, enquanto Junge (15) estimou que, durante os jogos olímpicos de 2004, houve um índice de 0,65 lesões por partida de basquetebol (ou 65 lesões a cada 1000 partidas). Têm-se na literatura evidências apontando que alterações no padrão de movimento de corredores correspondem a uma das causas associadas a fracturas por estresse e outras lesões por excesso de treinamento $(7,21,14,27)$, no entanto relações similares precisam de ser melhor determinadas no basquetebol. Valiant e Cavanagh (28) afirmam que forças aplicadas em grande magnitude e repetidamente podem representar uma condição suficientemente crítica para o desenvolvimento de lesões por estresse nos jogadores de basquetebol.

Um outro factor que necessita de mais estudos diz respeito à influência do desempenho do salto na determinação da sobrecarga gerada nas aterrissagens. Em estudo sobre a influência do uso de instruções relacionadas à cinemática das articulações para redução do impacto, McNair et al. (20) concluíram que técnicas de aterrissagem mais eficientes resultam em atenuações na magnitude da força de reação do solo (FRS). Hoffman et al. (12), concluíram que a diferença na FRS encontrada na aterrissagem de saltadores experientes e inexperientes pode ser devido à utilização mais eficiente da capacidade de atenuação de carga por parte da musculatura esquelética. Para que uma eventual relação entre as demandas do basquetebol e a incidência de lesões possa ser estabelecida, são necessários estudos preliminares sobre as características de dados dinâmicos dos movimentos usuais do jogo. Neste contexto, este estudo teve como objectivo central determinar características de parâmetros da FRS em seis movimentos típicos do basquetebol: arremesso em suspensão (jump), salto com contramovimento (jump \& reach), bandeja, rebote e corrida com bola rápida e lenta, em jogadores brasileiros profissionais. Teve-se como objetivo específico do estudo a análise da influência do desempenho do salto nas características da carga gerada nas aterrissagens.

\section{MATERIAIS E MÉTODOS}

\section{Amostra}

Considerando o interesse em estudar atletas profissionais, a amostra foi seleccionada intencionalmente dentre os jogadores que integravam a seleção brasileira de basquetebol. Como membros da selecção, os voluntários participaram do Torneio Pr é -Olimpico e do Campeonato Brasileiro de Basquete no ano de 2003. Atletas que nos últimos seis meses tivessem sofrido lesões ósteo-mio-articulares que os incapacitasse a participar de sua rotina de treinamento e 
competição, foram excluídos da amostra. A avaliação da condição ósteo-mio-articular do aparelho locomotor e do histórico de lesões foi determinada por intermédio de exames clínicos e anamnese ortopédica. Em função dos critérios de inclusão estabelecidos, a amostra foi composta por oito jogadores. Os voluntários seleccionados para o estudo apresentam $24 \pm 2,8$ anos de idade, $92 \pm 13,5 \mathrm{~kg}$, e estatura de $1,96 \pm 0,09 \mathrm{~m}$. Todos foram informados acerca dos propósitos e procedimentos adoptados e assinaram um termo de concordância, no qual afirmaram estarem cientes e de acordo com os procedimentos empregados no estudo.

\section{Instrumentos}

Para determinação das variáveis dinâmicas dos movimentos selecionados, utilizou-se uma plataforma de força (KISTLER AG, 9287 A). Esta plataforma possui transdutores de força do tipo piezoelétricos localizados nos cantos da superfície de medição $(0,6 \times 0,9 \mathrm{~m})$. Os sinais obtidos pelos transdutores são enviados por intermédio de cabos e interruptores a um amplificador de sinais (KISTLER AG, 9865 B), programados automaticamente para obtenção dos valores das três componentes da força de reacção do solo. O controle sobre a aquisição, análise e armazenamento dos dados foi realizado pelo programa de funções BIOWARE (282A1-20). Na execução das colectas utilizou-se frequência de amostragem igual a $1 \mathrm{kHz}$.

\section{Variáveis analisadas}

As variáveis dinâmicas da FRS seleccionadas para a análise dos saltos e da corrida encontram-se descritas nas Tabelas 1 e 2 e representadas graficamente nas Figuras 1 e 2.

Tabela 1. Variáveis analisadas para os movimentos de salto.

\begin{tabular}{|c|c|}
\hline Variáveis & Símbolo \\
\hline a) Menor valor da força vertical na decolagem (PC) & Fy Min \\
\hline b) Maior valor da força vertical na decolagem (PC) & Fy max D \\
\hline c) Tempo para atingir o Fy máximo na decolagem (s] & $\Delta \mathrm{t} F y \max \mathrm{D}$ \\
\hline d) Impulso para a decolagem (PC.s ] & Imp_D \\
\hline e) Tempo de fase aérea (s) & $\Delta t \mathrm{f}$ aer \\
\hline f) Maior valor da força vertical na aterrissagem (PC) & Fy max_A \\
\hline g) Tempo pa ra atingir o Fy máximo na aterrissagem (s) & $\Delta \mathrm{t}$ Fy max $\mathrm{A}$ \\
\hline h) Impulso nos primei ros 75 ms da aterrissagem (PC.s) & Imp_75 \\
\hline i) Altura dos saltos (m) & Alt sa lto \\
\hline
\end{tabular}

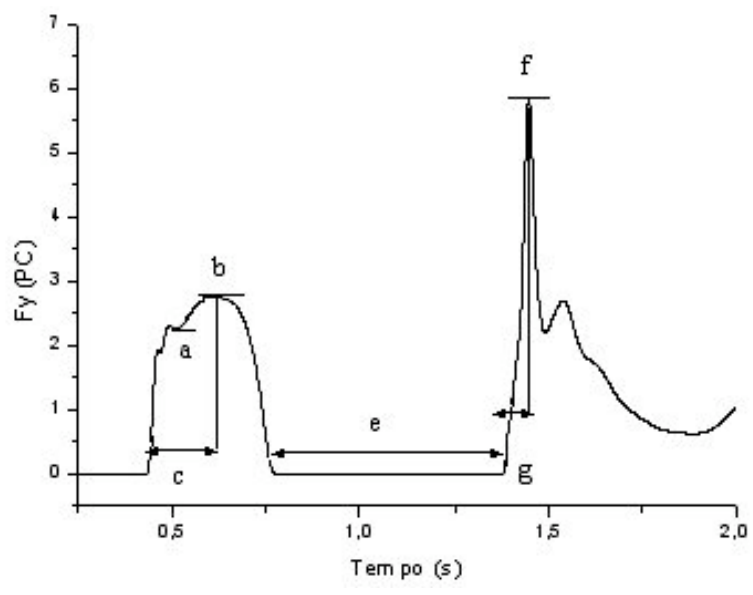

Figura 1. Curva representativa dos parâmetros da componente vertical da FRS seleccionados para a análise, para o movimento da bandeja.

Tabela 2. Variáveis analisadas para os movimentos de deslocamento.

\begin{tabular}{ll}
\hline Variáveis & Símbolo \\
\hline a) Primei ropico da força vertical (PC) & Fy max 1 \\
b) Tempo para atingir Fy max 1 (s) & $\Delta t$ Fy max 1 \\
c) Segundo pico da força vertical (PC) & Fy max 2 \\
d) Tempo para atingir Fy max 2 (s) & $\Delta t$ tFy max 2 \\
e) Impulso nos primeiros 75 ms da aterrissagem (PC.s) & Imp_75 \\
f) Maior magnitude da força de frenagem (PC) & Fx min \\
g) Tempo para atingir Fx mínima (s) & $\Delta$ tFx min \\
h) Maior magnitude força de propulsão (PC) & Fx max \\
i) Tempo para atingir Fx máxima (s) & $\Delta$ tFx max \\
j) Impulso de frenagem (PC.s] & Imp_fren \\
I) Impulso de Propulsão (PC.s) & Imp_prop \\
\hline
\end{tabular}

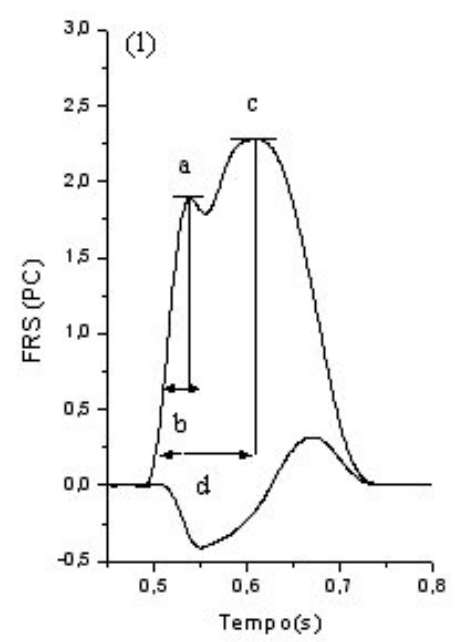




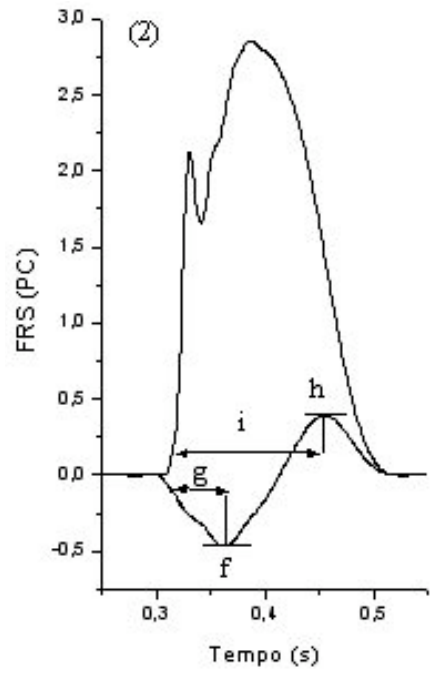

Figura 2. Curva representativa dos parâmetros seleccionados das componentes vertical e horizontal da FRS para os deslocamentos com bola. A figura (1) representa a corrida lenta, a uma velocidade média de $4 m . s^{-1}$ e a figura (2) representa a corrida rápida, a uma velocidade média de $6 \mathrm{~m} \cdot \mathrm{s}^{-1}$.

Os principais indicadores das cargas externas podem ser obtidos pela análise da componente vertical da FRS. Entre eles, o primeiro pico de força (Fy max_A) e o tempo para alcançar Fy max_A ( $\Delta$ t Fy max_A) são de reconhecida relevância para análise dos movimentos por permitirem a quantificação do impacto aplicado ao aparelho locomotor (2). Considerando que a resposta muscular dos membros inferiores não ocorre antes de $40 \mathrm{~ms}$ (32), optou-se por determinar o impulso nos primeiros 75 ms (Imp_75), como parâmetro indicador da carga externa aplicada num momento crítico para o aparelho locomotor.

Dessa forma, pode-se dizer que as variáveis da FRS referentes à aterrissagem dos saltos servem como indicadores da carga mecânica dos saltos, enquanto que a altura do salto e as variáveis referentes à decolagem servem como parâmetros indicadores do desempenho do salto.

A altura dos saltos (Alt_salto) foi determinada através da fórmula:

$h=\frac{(1 / 2 \pi V \cdot g)^{2}}{2 g}$, onde:

$\mathrm{h}=$ Altura em metros;

$\mathrm{TV}=$ Tempo de vôo em segundos;

$\mathrm{g}=$ aceleração da gravidade $\left(9,81 \mathrm{~m} . \mathrm{s}^{2}\right)$
Apesar da força reactiva médio-lateral ter sido colectada, não foram evidenciados resultados significantes, uma vez que no protocolo não foram executados movimentos com mudanças bruscas de direcção. Dessa forma, optou-se pela omissão desses dados.

\section{Procedimento Experimental}

Os movimentos seleccionados para analise foram o arremesso em suspensão (jump), a bandeja, o rebote e o deslocamento com bola a duas velocidades (4 e $\left.6 \mathrm{~m} . \mathrm{s}^{-1}\right)$. Tais movimentos foram seleccionados em função da importância que desempenham na consecução das acções ofensivas e defensivas que caracterizam o Basquete. Além destes que são movimentos típicos do basquete, optou-se por analisar ainda o salto com contramovimento (jump\&reach), que apesar de não compor os movimentos fundamentais do basquete, é um bom indicador das características do salto dos jogadores (6). Todas as colectas de dados foram realizadas em ambiente de laboratório. Objectivando a redução da potencial influência que a ausência de importantes referenciais de jogo, como a tabela e as marcações de quadra, poderiam exercer na realização dos movimentos a serem analisados, buscou-se a adequação do ambiente no qual a colecta dos dados foi realizada. Para tanto, delimitou-se uma área com as mesmas dimensões do garrafão no qual foi montada, também em acordo com as dimensões oficiais, uma tabela. A plataforma de força encontrava-se fixada no chão, e a tabela era movimentada de acordo com o movimento realizado. Ainda que tais adaptações não tenham permitido reproduzir com a necessária fidelidade as características de uma quadra de basquete, pode-se considerar que os voluntários executaram os movimentos planejados numa condição bastante próxima da condição real.

Para a execução dojump solicitou-se que o voluntário executasse o arremesso na linha de lance livre. Foram analisados apenas os dados relativos aos arremessos bem sucedidos. Para a execução do rebote, o voluntário era instruído a retomar a posse de uma bola arremessada por um auxiliar na altura do aro. Para tanto solicitava-se que o voluntário executasse o salto na sua expressão máxima de rendimento. Foram consideradas apenas as tentativas nas quais o voluntário conseguiu recuperar a posse de bola. A bandeja foi executada a partir da recepção da bola, passada por um auxiliar, para o voluntário já em movimento. A bola era recebi- 
da na proximidade da área de lance livre para que o voluntário pudesse realizar a bandeja. Foram avaliadas apenas as tentativas que resultaram em arremessos bem sucedidos. A condução de bola foi realizada em direcção a tabela, com uma distância mínima de partida da ordem de $10 \mathrm{~m}$. Os voluntários foram instruídos a realizar o movimento em duas velocidades, uma representando sua velocidade típica de condução de bola em situação de armação de jogadas $\left(4 \mathrm{~m} \cdot \mathrm{s}^{-1}\right)$. e a outra numa condição típica de contra ataque $\left(6 \mathrm{~m} \cdot \mathrm{s}^{-1}\right)$. OJump\&Reach foi executado estando o voluntário com as mãos na cintura. Após o comando o voluntário deveria flexionar os segmentos do membro inferior, caracterizando um movimento de preparação que antecede o salto final. Solicitou-se ao voluntário executar o salto empregando a sua maior capacidade de geração de força.

Todos os voluntários executaram todos os movimentos seleccionados para análise até se atingisse um número mínimo de dez tentativas válidas. A ordem de execução dos movimentos foi determinada por intermédio de sorteio. Os movimentos foram executados sem a presença de nenhuma espécie de marcação adversária. Durante a realização da colecta de dados, os voluntários utilizaram os calçados esportivos que usualmente utilizam em suas rotinas de treinamento e competição. Apesar de serem de marcas distintas, as características de construção dos calçados utilizados eram acentuadamente semelhantes.

\section{Tratamento dos dados e análise estatística}

Para eliminar ruídos provenientes de factores externos aos movimentos realizados, os dados provenientes da plataforma de forças foram filtrados através de um filtro butterworth recursivo de $2^{\mathrm{a}}$. ordem passabaixa de $40 \mathrm{~Hz}$.

Após a normalização dos dados pelo peso corporal de cada atleta, esses foram submetidos a um tratamento estatístico através do programa Statistica (versão 5.1, StatSoft, Inc.). A estatística descritiva foi baseada na apresentação dos valores médios e respectivos desviospadrão para cada movimento realizado. Através de uma correlação de Pearson, realizou-se as correlações entre a altura dos saltos e as variáveis indicadoras da carga mecânica.

\section{RESULTADOS E DISCUSSÃO \\ Movimentos de salto}

Os valores médios de pico da componente vertical da FRS relatados nos movimentos de salto do basquetebol se assemelham aos encontrados por McClay et al. (19), que oscilam de 2,7 vezes o peso corporal (PC) a 8,9 PC. Os valores médios de pico obtidos para a componente vertical da FRS nos movimentos de salto (Tabela 3, Figura 3), oscilam entre os 2,7 PC na decolagem do jump \& reach aos 7,27 PC na aterrissagem da bandeja. O valor mais alto do impulso $75 \mathrm{~ms}(0,24$ Peso Corporal segundo - PC.s), também foi encontrado na bandeja. O rebote apresentou as maiores magnitudes médias para o Impulso para a decolagem $(0,90$ PC.s) e o jump apresentou os menores valores médios (0.55 PC.s).

Esses valores (Tabela 3) correspondem a uma carga externa que se pode caracterizar como de média magnitude, levando em consideração a carga mecânica relatada na literatura $(1,24,25,30)$ em outras actividades, como a marcha (1,2 PC), a corrida (2,3 PC), o salto em altura (11 PC) e o salto triplo (20 PC).

\footnotetext{
Tabela 3. Média e desvio-padrão dos parâmetros relativos à componente vertical da Força de Reacção do Solo para os movimentos jump and reach, jump, rebote e bandeja, para os oito voluntários estudados. Os picos de força são apresentados na unidade peso corporal (PC), os intervalos de tempo em segundos [ $\mathrm{s}$ ], e a altura dos saltos em metros ( $\mathrm{m}$ ).
}

\begin{tabular}{lcccc}
\hline Variáveis & \multicolumn{3}{c}{ Movimentos analisados } \\
\hline & Jump \& Reach & Jump & Rebote & Bandeja \\
\hline Fy max_A (PC) & $5,05 \pm 0,87$ & $4,54 \pm 1,10$ & $5,39 \pm 1,26$ & $7,27 \pm 2,71$ \\
$\Delta$ t Fy max_A (s) $_{\text {Imp_75 (PC.s) }}$ & $0,07 \pm 0,04$ & $0,08 \pm 0,01$ & $0,07 \pm 0,01$ & $0,08 \pm 0,13$ \\
Fy Min (PC) & $0,17 \pm 0,03$ & $0,13 \pm 0,04$ & $0,18 \pm 0,04$ & $0,24 \pm 0,04$ \\
Fy max_D (PC) & $0,45 \pm 0,24$ & $0,54 \pm 0,20$ & - & - \\
$\Delta$ tFymax_D (s) & $2,7 \pm 0,42$ & $2,96 \pm 0,32$ & $3,19 \pm 0,78$ & - \\
Imp_prop (PC.s) & $0,43 \pm 0,09$ & $0,34 \pm 0,06$ & $0,46 \pm 0,22$ & - \\
Alt_salto (m) & $0,7 \pm 0,11$ & $0,55 \pm 0,07$ & $0,90 \pm 0,19$ & $0,61 \pm 0,04$ \\
\hline
\end{tabular}


(a)

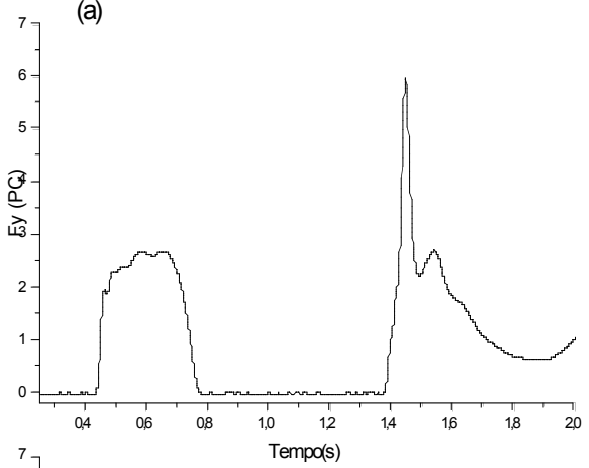

(c)

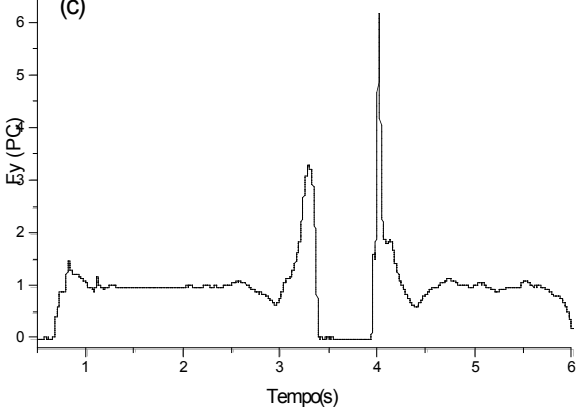

(b)

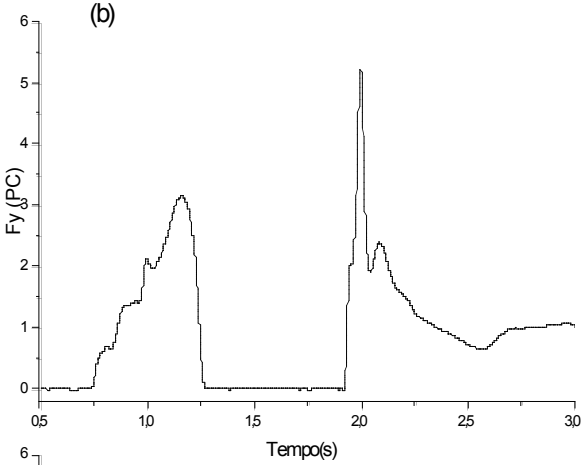

(d)

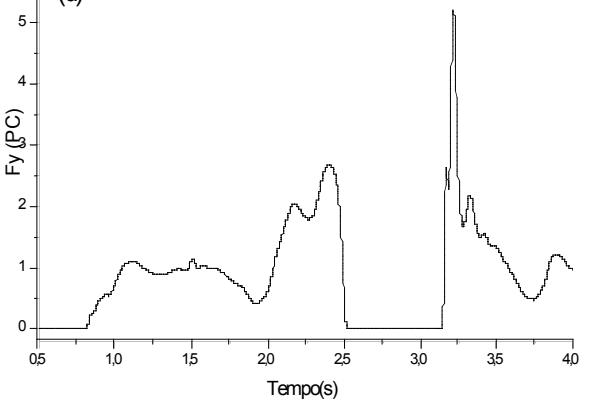

Figura 3. Curva representativa da componente vertical da FRS para o movimento da bandeja (a), do rebote (b), do jump (c) e do jump \& reach (d).
Uma possível relação entre essas forças externas, caracterizadas como de média magnitude, e o índice de lesões, pode ser estabelecida levando-se em consideração a frequência em que o aparelho locomotor dos atletas é submetido a elas. Além disso, torna-se importante ressaltar que é altamente provável que em situações reais de jogo a carga seja maior do que as reportadas, dada a dificuldade de motivar atletas competitivos a desempenhar os movimentos a níveis máximos em situação não competitiva.

\section{Deslocamentos com bola}

Os valores médios da componente vertical da FRS encontrados para os movimentos de corrida com bola (Tabela 4) são similares às magnitudes reportadas na literatura (24). Para a corrida rápida com bola $\left(6 \mathrm{~m} \cdot \mathrm{s}^{-1}\right)$ encontrou-se o valor médio de 2,16 PC para o primeiro pico da componente vertical da FRS (Fy max1) e o tempo para o pico (DtFy max1) foi de 0,03s. O valor do Imp_75 foi de 0,10 PC.s. Para a componente horizontal antero-posterior $(\mathrm{Fx})$, encontrou-se um pico de desaceleração (Fx min) de 0,48 PC e o tempo para esse pico foi de $0,05 \mathrm{~s}$. O impulso de frenagem foi de 0,02 PC.s.

Na corrida lenta $\left(4 \mathrm{~m} \cdot \mathrm{s}^{-1}\right)$, os valores médios para Fy max1, DtFy max 1 e Imp_75 foram, respectivamente, 1,85 PC, 0,03s e 0,09 PC.s. Para a Fx min e DtFx min, encontrou-se valores médios de 0,42 PC e 0,06 s. O impulso de frenagem foi de 0,02 PC.s. As variáveis acima citadas indicam as condições de desaceleração do membro inferior e a consequente transmissão do choque mecânico ao aparelho locomotor durante a corrida. A analise dos dados e consequente comparação com dados da literatura $(9,19,24)$ evidenciou que a condução de bola não afectou de forma significativa nenhuma dessas variáveis.

Os valores médios encontrados para as variáveis indicadoras dos eventos relacionados à fase propulsiva na corrida com bola rápida $\left(6 \mathrm{~m} . \mathrm{s}^{-1}\right)$ foram: $2,48 \mathrm{PC}$ para o segundo pico da força vertical (Fy max2), 0,09s para o tempo para o pico (DtFy max2), 0,35 PC para o pico da componente antero-posterior (Fx Max), 0,17s para o tempo para esse pico (DtFx Max) e de 0,02 PC.s para o impulso de propulsão (Imp_prop). Para a corrida com bola lenta $\left(4 \mathrm{~m} . \mathrm{s}^{-1}\right)$, os valores médios para Fy max2, DtFy max2, Fx Max, DtFx Max e Imp_prop foram, respectivamente, 2,52 PC, 0,11s, 0,30 PC, 0,19s e 0,02 PC.s. 
Esses resultados sugerem que, bem como as variáveis relacionadas à desaceleração do membro inferior, as variáveis relacionadas à fase propulsiva da corrida também não foram influenciadas pela condução da bola, evidenciando que tanto o controle do choque mecânico quanto a produção de força propulsiva não foram afectadas pela execução da tarefa em questão.

Tabela 4. Média e desvio-padrão dos parâmetros relativos à componente vertical e ântero-posterior da Força de Reacção do Solo para a corrida rápida [ $v=6 m \cdot s^{-1}$ ] e lenta com bola $\left(v=4 m \cdot s^{-1}\right.$ ] para os oito voluntários estudados. Os picos de força são apresentados na unidade peso corporal [PC], os intervalos de tempo em segundos [s].

\begin{tabular}{lcc}
\hline Variáveis & \multicolumn{2}{c}{ Movimentos analisados } \\
\hline & $\begin{array}{c}\text { Corrida Rápida } \\
\text { com Bola }\end{array}$ & $\begin{array}{c}\text { Corrida Lenta } \\
\text { com Bola }\end{array}$ \\
\hline Fy max 1(PC) & $2,16 \pm 0,37$ & $1,85 \pm 0,28$ \\
$\Delta \mathrm{tFy} \max 1(\mathrm{~s})$ & $0,03 \pm 0,008$ & $0,03 \pm 0,009$ \\
Fy max 2 (PC) & $2,48 \pm 0,37$ & $2,52 \pm 0,42$ \\
$\Delta \mathrm{tFy} \max 2$ (s) & $0,09 \pm 0,02$ & $0,11 \pm 0,02$ \\
Imp_75 (PC.s) & $0,10 \pm 0,02$ & $0,09 \pm 0,02$ \\
Fx min (PC) & $0,48 \pm 0,14$ & $0,42 \pm 0,10$ \\
$\Delta \mathrm{tFx} \min (\mathrm{s})$ & $0,05 \pm 0,01$ & $0,06 \pm 0,01$ \\
Fx max (PC) & $0,35 \pm 0,08$ & $0,30 \pm 0,12$ \\
$\Delta \mathrm{tFx} \max (\mathrm{s})$ & $0,17 \pm 0,03$ & $0,19 \pm 0,04$ \\
Imp_fren (PC.s) & $0,02 \pm 0,01$ & $0,02 \pm 0,005$ \\
Imp_prop (PC.s) & $0,02 \pm 0,003$ & $0,02 \pm 0,004$ \\
\hline
\end{tabular}

\section{Correlação entre o desempenho do salto e a FRS} na aterrissagem

Conforme considerado anteriormente, outro objectivo desse estudo foi determinar a influência do desempenho do salto, traduzido pela altura do salto, na determinação da sobrecarga externa gerada nas aterrissagens.

Nesse sentido, é interessante destacar que a aterrissagem da bandeja, embora correspondesse à maior média de magnitude de pico da força reactiva vertical $(7,27 \pm 2,71 \mathrm{PC})$ e do $\operatorname{Imp} 75$ (0,24 $\pm 0,04$ PC.s), não resultou de saltos com as médias mais elevadas das alturas atingidas $(0,44 \pm 0,02 \mathrm{~m}$ - Tabela 3$)$, condicionando a baixa correlação encontrada $(r=0,34 \mathrm{e}$ $0,44)$ entre as duas variáveis e a altura do salto (Tabela 5). Esse fato evidencia uma considerável independência entre indicadores de carga e a altura de queda.
As correlações (r) entre a altura do salto no jump $\mathcal{E}$ reach e a Fy max_A e Imp 75 foram, respectivamente, 0,36 e 0,58. Para a altura do jump, as correlações foram de 0,44 para a Fy max_A e de 0,62 para o Imp 75. No rebote, as correlações entre a altura do salto e Fy max_A e o Imp 75 foram de 0,22 e 0,43, respectivamente.

Tabela 5. Correlação ( $r$ ] entre altura dos saltos realizados e as variáveis indicadoras da carga e do rendimento $\left({ }^{*} p<0,05,{ }^{* *} p<0,001\right)$.

\begin{tabular}{lcccc}
\hline & $\begin{array}{c}\text { Altura salto } \\
\text { no jump\&reach }\end{array}$ & $\begin{array}{c}\text { Altura salto } \\
\text { no jump }\end{array}$ & $\begin{array}{c}\text { Altura salto } \\
\text { no rebote }\end{array}$ & $\begin{array}{c}\text { Altura salto } \\
\text { na bandeja }\end{array}$ \\
\hline Fy max_A & $0,36^{*}$ & $0,44^{* *}$ & 0,22 & 0,34 \\
Imp 75 & $0,58^{*}$ & $0,62^{* *}$ & $0,43^{*}$ & 0,44 \\
\hline
\end{tabular}

Os dados evidenciam que a baixa correlação encontrada para o movimento da bandeja também ocorre para os outros movimentos seleccionados para o estudo (Tabela 5), fato que corrobora a ideia de que a técnica de movimento possui maior influência na atenuação das forças externas do que a altura de queda isoladamente. O estudo de Zhang et al. (34) suporta esta hipótese. Os resultados apresentados por estes autores sugerem que os extensores do joelho desempenham importante papel na atenuação da sobrecarga mecânica gerada durante as aterrissagens.

\section{CONCLUSÕES}

Os resultados obtidos evidenciam que o aparelho locomotor experiencia cargas relativamente altas durante a realização dos movimentos típicos do basquetebol, fato que, quando associado à frequência de exposição dos atletas, pode explicar as lesões características da modalidade. Deve-se considerar que um melhor entendimento acerca desta questão ainda depende de uma análise do volume de aplicação destas cargas.

Foi verificado, também, que o desempenho do salto não possui influência determinante na carga gerada nas aterrissagens, o que nos permite concluir que a estrutura de movimento pode desempenhar papel de destaque no controle das forças externas geradas nas aterrissagens. Novos estudos são necessários para identificar as características cinemáticas, dinâmicas e eletromiográficas empregadas nessa condição de controle. 
NOTA

${ }^{1}$ Médico do time de basquete norte-americano Washington Bullets.

\section{CORRESPONDÊNCIA}

\section{Fernanda Michelone Acquesta}

Rua do Manifesto, 2810 - Ipiranga

CEP 04209-003 São Paulo SP, Brasil

ou

Laboratório de Biomecânica da Escola de Educação

Física e Esporte da Universidade de São Paulo

Av. Mello Moraes, 65 - Cidade Universitária

CEP 05508-900 São Paulo SP, Brasil

e-mail: acquesta@usp.br

\section{REFERÊNCIAS BIBLIOGRÁFICAS}

1. Amadio AC (1989). Fundamentos da biomecânica do esporte: considerações sobre a análise cinética e aspectos neuromusculares do movimento. São Paulo. 119p. Tese (Livre Docência) Escola de Educação Física, Universidade de São Paulo.

2. Amadio AC, Duarte M (1996). Fundamentos biomecânicos para análise do movimento humano. São Paulo: Laboratório de Biomecânica/EEFEUSP

3. Amadio AC, Serrão JC (1997). Instrumentação em cinética. In: SAAD, M. Análise da marcha, manual do CAMOSBMFR, São Paulo.

4. Arena SS (2005). Estudo epidemiológico das lesões esportivas no basquetebol, futsal e voleibol ocorridas em atletas jovens. $125 \mathrm{p}$. Tese (Doutorado). Faculdade de Medicina, Universidade de São Paulo, São Paulo.

5. Aplle DF (1988). Basketball injuries: An overview. Phys Sports Med 16(12): 64-74

6. Ávila AO, Amadio AC, Guimarães ACS, David AC, Mota CB, Borges DM, Guimarães FJSP, Menzel HJ, Carmo J, Loss JF, Serrão JC, Sá MR, Barros RML (2002). Métodos de medição em Biomecânica do esporte: descrição de protocolos para aplicação nos centros de excelência esportiva. Revista Brasileira de Biomecânica 3(4):57-67

7. Bates BT, Osternig LR, Mason B, James LS (1979). Foot orthotic devices to modify selected aspects of lower extremity mechanics. Am J Sports Med 7(6):338-42

8. Colliander E, Eriksson E, Herkel M (1986). Injuries in Swedish elite basketball. Orthopedics 9(2):225-27

9. Crossley K, Bennell KL, Wrigley T, Oakes BW (1999). Ground reaction forces, bone characteristics, and tibial stress fracture in male runners. Med Sci Sports Exerc $31(8): 1088-93$

10. Harmer PA (2005). Basketball injuries. Med Sports Sci 49:31-61

11. Henry JH, Lareau B, Neigut D (1982). The injury rate in professional basketball. Am J Sports Med 10:16-18

12. Hoffman JR, Liebermann D, Gusis A (1997). Relationship of leg strength and power to ground reaction forces in both experienced and novice jump trained personnel. Aviat Space Environ Med 68(8):710-14

13. Iwamoto J, Takeda T (2003). Stress fractures in athletes: review of 196 cases. J orthop Sci 8(3):273-78

14. James SL (1995). Running injuries to the knee. J Am Acad of Orthop Surg 3(6):309-18

15. Junge A Langevoort G, Pipe A, Peytavin A, Wong F, Mountjoy M, Beltrami G, Terrell R, Holzgraefe M, Charles $\mathrm{R}$, Dvorak J. (2005). Injuries in team sport tournaments during the 2004 Olympic Games. Am J Sports Med 3(4):565-76

16. Major NM (2006). Role of MRI in prevention of metatarsal stress fractures in collegiate basketball players. $\mathrm{Am} \mathrm{J}$ Roentgenol 186: 255-58

17. McBryde AM Jr (1985). Stress fractures in runners. Clin Sports Med 4(4): 737-52

18. McClay IS, Robinson JR, Andriacchi TP, Frederick EC, Gross T, Martin P, Valiant G, Williams KR, Cavanagh PR (1994a). A Kinematic profile of skills in professional basketball players. J Appl Biomech 10: 205-21

19. McClay IS, Robinson JR, Andriacchi TP, Frederick EC, Gross T, Martin P, Valiant G, Williams KR \& Cavanagh PR (1994b). A profile of ground reaction forces in professio- 
nal basketball players. J Appl Biomech 10: 222-36

20. McNair PJ, Prapavessis H, Callender K (2000). Decreasing landing forces: effect of instruction. Br J Sports Med 34(4): 293-96

21. Messier SP, Pittala KA (1988). Etiologic factors associated with selected running injuries. Med Sci Sports Exerc 20(5): 501-05

22. Miller S, Bartlett RM (1993). The effects of increased shooting distance in the basketball jump shot. J Sports Sci 11(4): 285-93

23. Miller S, Bartlett RM (1996). The relationship between basketball shooting kinematics, distance and playing position. Journal of Sports Sciences 14(3): 243-53

24. Munro CF, Miller DI, Fuglevand AJ (1987). Ground reaction forces in running: A Reexamination. J Biomech 20(3): 147-55

25. Rab GT (1994). Human locomotion. In: Rose J; Gamble JG, Human walking. Baltimore, Willians \& Wilkings, 101-21

26. Reeder MT, Dick BH, Atkins JK, Pribis AB, Martinez JM. (1996). Stress fractures. Current concepts of diagnosis and treatment. Sports Med 22(3): 198-212

27. Stergiou N, Bates BT, James SL. (1999). Asynchrony between subtalar and knee joint function during running. Medicine and Science in Sports and Exercise 31(11): 1645-55

28. Valiant GA, Cavanagh PR (1987). A study of landing from a jump: implications for the design of a basketball shoe. In: RW Norman, RP Wells, KC Hayes, \& AE Patta. Biomechanics XB. Champaign, IL. Human Kinetics, 117-22

29. Warren BL (1990). Plantar fasciitis in runners. Treatment and prevention. Sports Med 10(5): 338-45

30. Winter DA (1990). Biomechanics and Motor Control of human movement. John Wilwey \& Sons Inc., New York, Chichester, Brisbane, Toronto, Singapore.

31. Winter DA (1991). The biomechanics and motor control of human gait: normal, elderly and pathological. $2^{\text {a }}$ ed. University of Waterloo Press

32. Winter DA, Bishop PJ (1992). Lower extremity injury. Biomechanical factors associated with chronic injury to the lower extremity. Sports Med 14(3): 149-156.

33. Zelisko JA, Noble HB, Porter M (1982). A comparison of men's and women's professional basketball injuries. Am J Sports Med 10(5): 297-99

34. Zhang S, Clowers K, Kohstall C, Yu YJ (2005). Effects of various midsole densities of basketball shoes on impact attenuation during landing activities. J Appl Biomech $21(1): 3-17$ 